\title{
Experts predict bleak post-genomic era for drug R\&D
}

With the 3.2 gigabase human genome sequenced and mapped to such a high degree, scientists are not the only people turning their focus on the post-genomic era. The pharmaceutical and biotechnology analyst house, Lehman Brothers, has paired up with management consultants McKinsey to assess the impact of genomics on drug development. Among their conclusions is the finding that the plethora of new drug targets that the sequencing effort should yield will, in fact, increase overall $R \& D$ costs so acutely over the next five years that it may bankrupt some pharmaceutical companies.

Over the past century, the pharmaceutcal industry has been dependent on a mere 483 drug targets for which to develop new medicines, but experts estimate that the human genome sequence will increase that number by several thousand. The Lehman/McKinsey report concludes that a threshold investment of US \$70-100 million in new genomic technologies is required by companies wanting to profit from this knowledge, but thereafter, increased spending does not result in increased return.

Once targets have been identified by methods such as database mining, microarray analysis and single nucleotide polymorphism profiling-93\% of genes contain a SNP-companies require technology to validate drug targets, and ac- cording to Lehman/McKinsey it is this stage that presents the weakest short-term link.

For example, although gene knockouts and other model organisms are now a common research tool, the interviews conducted with experts to draw up the report lead Lehman/McKinsey to conclude that they are only of modest value "since the phenotypes are consistent across species only half the time." Technologies that look at proteins and not genes are now the focus of most excitement within the R\&D industry.

Their report states that validation technologies such as functional genomics are not presently robust enough to add value to the R\&D pipeline: "The flood of new targets has not been matched with a flood of biological information about these targets. While sequencing has become industrialized, target validation is still very much a craft."

In the times of traditional pharmaceutical R\&D-that is, up until 1995-companies had an average of 50 targets per year under evaluation, and drugs of a similar class were already on the market for $70 \%$ of these targets. From now unt 2035 , around 200 targets per year are anticipated, of which $70 \%$ will be 'unprecedented', meaning that their functional biology has not been tested before. Unprecedented targets also require new chemistries that have yet to be developed.

Because toxicology and drug suitability (administration, distribution, metabolism and excretion; $\mathrm{ADME}$ ) are often determined at the medicinal chemistry stage, the lack of chemical ancestry for new compounds means that these drugs may progress as far as costly Phase II trials before adverse properties are picked up. This, says the report, is the key reason that $R \& D$ costs will rise in the short term. It is estimated that profits from a new compound will drop from $\$ 263$ million to $\$ 34$ million during this time.

A slight change in analysis gives a negative value for these programs, 'suggesting that he industry could go bankrupt trying to innovate."

Areas that will yield value beyond 2005 are "new toxicology modeling, better chemistries and patient stratification in clinical development." The report also predicts that the future of toxicology, ADME and chemical analysis of new compounds lies in informatics, such that screening for these properties will be computerized, thus saving time and money. And it concludes that while proteomics is "still in its infancy...experts we have consulted have confirmed that the value of proteomics will far exceed the value of sequencing the human genome."

Karen Birmingham, London

\section{Experiments are destined for space}

On 7 February, space shuttle Atlantis left Kennedy Space Center in Florida carrying a \$1.4 billion, state-of-the-art science laboratory. Called the Destiny laboratory, it will house equipment for measuring the effects of radiation and $\mathrm{mi}$ crogravity on humans, growing protein crystals for structural studies, and within the next five years, plant and animal experiments.

Destiny is the first of six planned research modules to be delivered to the International Space Station (ISS), a joint project of the United States, Russia, Japan, Europe and Canada, currently orbiting 230 miles above the earth. The Atlantis shuttle mission, scheduled to last 11 days, includes three space walks during which the astronauts will attach the Destiny laboratory to the space station.

Destiny, a 28-foot-long $(\sim 8.5 \mathrm{~m})$ cylinder, will be the most sophisticated and versatile laboratory ever used in space, according to NASA. It will be joined in space by other laboratory modules sponsored by the National Space Development Agency of Japan, the European Space Agency and Rosaviakosmos.

According to Dave Drachilis, NASA spokesperson at the Marshall Space Flight Center in Huntsville, Alabama, initial research onboard the Destiny module will focus on the effects of long-term interplanetary travel on humans. For example, a synthetic torso will measure the effects of radiation inside the space station on human organs. Other experiments will look at the effects of prolonged space flight on human skeletal muscle and bone because astronauts experience bone mass loss in space, which resembles osteoporosis but is re-

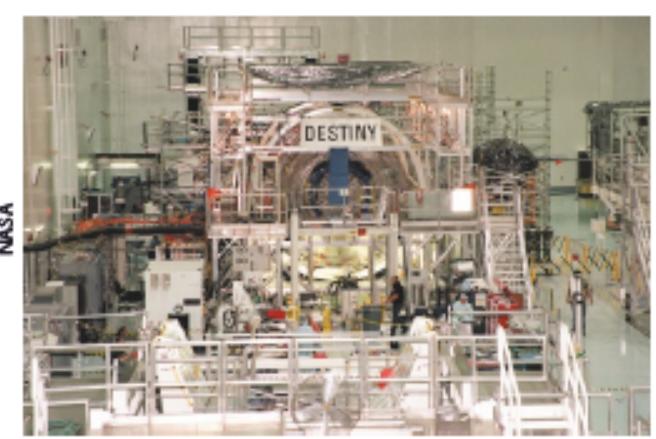

Construction of Destiny at the Kennedy Space Center, Florida 